\title{
Cross-Talk between Inducible Nitric Oxide Synthase and Cyclooxygenase in Helicobacter-pylori-Induced Gastritis
}

\author{
L. Franco ${ }^{\mathrm{a}}$ G. Talamini ${ }^{\mathrm{b}}$ \\ ${ }^{a}$ Section of Pharmacology, Department of Medicine and Public Health, and ${ }^{\mathrm{b}}$ Department of Surgical and \\ Gastroenterological Sciences, University of Verona, Verona, Italy
}

\author{
Key Words \\ Helicobacter pylori - Inducible nitric oxide synthase • \\ Cyclooxygenase $2 \cdot$ Gastric mucosa $\cdot$ Nitric oxide $\cdot$ \\ Prostaglandin
}

\begin{abstract}
Objectives: The present study examined the cross-talk between prostanoids and nitric oxide (NO) in human gastric biopsies during Helicobacter pylori infection. Subjects and Methods: A pool of 1 or 2 biopsies per patient $(11$ H. pylori positive and $9 \mathrm{H}$. pylori negative) were incubated in the medium with/without drugs, $1400 \mathrm{~W}$ and NS-398, inhibitors of inducible nitric oxide synthase (iNOS) and cyclooxygenase 2 $(\mathrm{COX}-2)$, respectively. Levels of $\mathrm{NO}$ and prostaglandin $\mathrm{E}_{2}$ $\left(\mathrm{PGE}_{2}\right)$, predominant products of activity of NOS and COX enzymes, were measured in the medium whereas the expressions of iNOS and COX protein, examined by Western blotting, were measured in the biopsies. Results: The 11 patients with $H$. pylori infection showed a marked expression of COX-2 and iNOS proteins and high levels of $\mathrm{PGE}_{2}$ and NO, as a consequence of iNOS and COX-2 activation, while proteins were absent and the level of nitrite and $\mathrm{PGE}_{2}$ was low in the 9 noninfected patients. The COX-2 inhibitor decreased both $\mathrm{NO}$ and $\mathrm{PGE}_{2}$. The iNOS-specific inhibitor decreased NO but did not have any effect on the increase in gastric mucosal $\mathrm{PGE}_{2}$. Both inhibitors had no effect on the protein level of these two enzymes. Conclusions: The data showed that COX-2 inhibitor might modulate the iNOS pathway, suggest-
\end{abstract}

ing that COX-2 activity and/or its products may be related to the functional activation of iNOS but not to the expression of iNOS protein.

Copyright $\odot 2009$ S. Karger AG, Basel

\section{Introduction}

The gastric mucosa is frequently exposed to various stimulants including acid, pepsin, alcohol, oxidative irritants, drugs and bacteria, resulting in gastric mucosal cell death by both necrosis and apoptosis [1]. The integrity of the gastrointestinal mucosa depends on the interplay of various protective and damaging factors to which this mucosa is exposed. The major lines of defence include the continuous mucus-alkaline secretion, the maintenance of the mucosal blood flow, and the restitution or proliferation of mucosal cells [2]. Although many chemical mediators participate in producing coordinated and effective mucosal responses to injury, increasing evidence suggests that endogenously produced nitric oxide (NO) maintains the gastric mucosal integrity in combination with prostanoids, including prostaglandins (PGs) [3]. NO and prostanoids are synthesized by nitric oxide synthase (NOS) and cyclooxygenase (COX), respectively, both of which have constitutive and inducible isoforms $[4,5]$. In the gastric mucosa, two types of constitutive NOS have been discovered, neuronal NOS and endothelial NOS $[6,7]$. Inducible NOS (iNOS) is expressed in the cells involved in 
inflammation and immune activation by stimulation with certain cytokines and/or endotoxins. NO is an important messenger, which not only plays an important physiological role in many systems and cells, but also contributes to the pathogenesis of many conditions, including cancer [8]. In the stomach, the constitutive form results in the production of physiological levels of NO important in gastric mucosal functions. When appropriately stimulated, such as after exposure to endotoxin, iNOS can also be detected $[9,10]$, and it produces larger quantities of NO that have been shown to contribute to gastric damage, particularly when cells are exposed to large quantities of NO over an extended period of time [11]. Paradoxically, it also contributes to mucosal integrity in some circumstances $[10,12]$. This can be illustrated by the ability of different NO concentrations to produce completely opposite effects in the same tissue [13]. COX is the rate-limiting enzyme for the production of PGs from arachidonic acid. COX-2 is the inducible form of the COX enzyme, the synthesis of which is induced rapidly and transiently by various proinflammatory mediators and mitogen stimuli. The predominant form in the stomach is COX-1, which is constitutively expressed and is involved in the production of PGs for cellular 'housekeeping' functions, including gastric cytoprotection. Although the gastric mucosal integrity in inflammatory states can be modulated by the concerted action of endogenous NO and PGs, little is known of the cross-talk effects between them. Helicobacter pylori infection is recognized as one of the most common chronic infections in humans, and its colonization of gastric epithelium result in chronic gastritis, gastric cancer and gastric B cell lymphoma [14]. H. pylori infection is one of the risk factors for gastric carcinogenesis due to a continuous formation of reactive nitrogen, including NO [15]. The cross-talk represents an important mechanism by which the initial inflammatory response can be amplified or attenuated. In order to understand the relationship between COX-2 and iNOS, we examined the effect of 1400W and NS-398, inhibitors of iNOS and COX2 , on the levels of $\mathrm{NO}$ and prostaglandin $\mathrm{E}_{2}\left(\mathrm{PGE}_{2}\right)$, a predominant product of activity of NOS and COX enzymes, and expression of iNOS and COX-2 proteins in gastritis.

\section{Subjects and Methods}

Subjects

A total of 20 subjects with dyspepsia undergoing upper gastrointestinal endoscopy were analysed. Informed consent was obtained from each enrolled patient. None of the patients had been on non-steroidal anti-inflammatory drugs, $\mathrm{H}_{2}$ receptor antagonists, proton pump inhibitors, bismuth compounds or antibiotics during the past 2 months. During endoscopy, gastric biopsy specimens were taken using autoclave-sterilized forceps from the antral mucosa for culture and histological examination, which was performed in a blinded manner. The biopsy specimens were fixed in $10 \%$ formalin and embedded in paraffin. Sections were stained with haematoxylin and eosin and Giemsa for detection of $H$. $p y$ lori. Helicobacter colonization was diagnosed by identification of characteristic curved or spiral bacilli. The following semiquantitative grading based on the Sydney system classification [16] was used for absence or presence of $H$. pylori: absence $=---$; focal presence of small amounts $=+--$; intermediate situation between small and large amounts $=++-$; diffuse presence of large amounts $=+++$.

\section{Gastric Biopsies}

The biopsy specimens of antral mucosa, 5 or 6 for each patient, were immediately washed twice with ice-cold phenol-red-free minimum essential medium and centrifuged at $3,000 \mathrm{~g}$ at $4^{\circ} \mathrm{C}$. A pool of 1 or 2 biopsies per patient was incubated at $37^{\circ} \mathrm{C}$ in $1 \mathrm{ml}$ of minimum essential medium containing $10 \%$ fetal bovine serum, penicillin (50 units $/ \mathrm{ml}$ ) and streptomycin $(0.5 \mu \mathrm{g} / \mathrm{ml})$ in a $5 \% \mathrm{CO}_{2}$ incubator. A COX-2-selective inhibitor, NS-398 (Cayman Chemical Co., Ann Arbor, Mich., USA; $10 \mu \mathrm{M}$ ) and a selective iNOS inhibitor, 1400W (Cayman; $5 \mu \mathrm{M}$ ) were added to each specimen. Following 24-hour incubation with/without drugs, supernatants were collected by aspiration and used for measuring nitrite and $\mathrm{PGE}_{2}$, and the biopsies were used for Western blot analysis.

\section{Nitrite and $P G E_{2}$ Determination}

The production of $\mathrm{NO}$ was assayed by measurement of the accumulation of nitrite $\left(\mathrm{NO}_{2}^{-}\right)$, the stable metabolite of $\mathrm{NO}$, in the culture medium. Nitrite accumulation in the culture supernatant was determined by first reducing the $\mathrm{NO}_{3}^{-}$using bacterial nitrate reductase prepared from Escherichia coli. Nitrite was assayed colorimetrically after reaction with the Griess reagent. All determinations were performed in duplicate. Nitrite concentrations were calculated by comparison with OD 550 of standard solutions of sodium nitrite in culture medium. Results were expressed as micromolars $\mathrm{NO}_{2}^{-}$.

$\mathrm{PGE}_{2}$ in the unextracted culture medium was measured by enzyme immunoassay (Cayman) according to the manufacturer's protocol [17]. Proteins from homogenized gastric biopsies were determined according to the method of Bradford [18]. The data were expressed as nanograms $\mathrm{PGE}_{2}$ per milligram of tissue protein per $24 \mathrm{~h}$. All the assays were performed in duplicate.

\section{Western Blot Analysis}

The levels of iNOS and COX-2 protein were examined by Western blotting. Gastric biopsies were homogenized in a cold lysis buffer containing: Tris, $50 \mathrm{mM}$, pH 8; EDTA, 10 mM; Triton-X 100, 1\%; leupeptin, $20 \mu \mathrm{g} / \mathrm{ml}$. Homogenates were centrifuged at $10,000 \mathrm{~g}$ for $15 \mathrm{~min}$ at $4^{\circ} \mathrm{C}$. The protein concentration of the supernatant was measured using the method of Bradford [18]. Supernatants with equal amounts of protein were subjected to electrophoresis $(125 \mathrm{~V}$ for $1.5 \mathrm{~h}$ with a Mini-Protean 3 Electrophoresis System, Bio-Rad Laboratories, Milano, Italy) on $10 \%$ for COX-2 or on $7.5 \%$ for iNOS sodium dodecyl sulphate polyacrylamide gel. The separated proteins were transferred to nitrocellulose (Schleicher \& Schuell, Dassel, Germany; $100 \mathrm{~V}$ for 1.5-2 h). The membrane was blocked overnight with non-fat dry milk 5\%, Tris-buffered saline (20 mM Tris, $137 \mathrm{mM} \mathrm{NaCl}, \mathrm{pH}$ 7.6), Tween-20 $0.1 \%$ and then incubated with the 
primary rabbit polyclonal antibody against human PGH synthase 2 (Cayman; 1:1,000 dilution) or against human iNOS (Santa Cruz Biotechnology; 1:200 dilution) for $1 \mathrm{~h}$. Purified mouse macrophage iNOS (Cayman) and purified ovine COX-2 (Cayman) were used as standard. After six washes, the blot was incubated (45 min) with an anti-rabbit immunoglobulin secondary antibody conjugated to horseradish peroxidase, from donkey (Amersham Pharmacia Biotech, Milano, Italy; 1:2,000 dilution). Bands were detected using the enhanced chemiluminescence detection system ECL (Amersham) and exposed to film (Hyperfilm ECL, Amersham) with hypercassette and then quantitated by densitometry (LKB Ultra Scan XL Laser densitometer). For internal control, the same membranes were washed, followed by incubation with mouse anti- $\beta$-actin for $1 \mathrm{~h}$, incubation with horseradish-peroxidase-linked goat antimouse IgG for $1 \mathrm{~h}$ and ECL visualization of bands. All other compounds were from Sigma Chemical Co. (St. Louis, Mo., USA).

\section{Statistics}

Data are expressed as mean \pm SEM. Statistical significance was determined by the Mann-Whitney $U$ test. A probability $p$ value of $\leq 0.05$ was taken to indicate statistically significant differences between groups.

\section{Results}

Of the 20 patients, 11 with positive results for $H$. $p y$ lori showed severe chronic active antral gastritis associated with duodenal ulcer, and all 9 patients with negative results for $H$. pylori showed normal mucosa based on gastrointestinal endoscopy and histology. Of the 11 patients positive for $H$. pylori, 5 were male and 6 female. Of the 9 negative ones, 5 were male and 4 female. The endoscopic findings in the patients were as follows: duodenal ulcer, 6; duodenal or prepyloric erosion, 2 samples; stenosis or deformed pylorus, 1 ; oesophagitis, 2 . The 9 negative samples had histologically non-inflamed antral mucosa and normal endoscopy. The $H$. pylori status of the positive patients ranged from moderate $(-++)$ to severe $(+++)$.

The data indicated that iNOS protein, assessed by Western blot analysis, is expressed in all gastric mucosae of patients with $H$. pylori infection and active gastritis. A representative Western blot profile of iNOS protein levels in human antral biopsy is shown in figure 1. Expression of iNOS protein was absent in all gastric mucosa samples of patients without $H$. pylori infection and with normal mucosa (fig. 1a, lane 2). The band of iNOS was also undetectable after addition of drugs before the incubation in all the samples. The gastric mucosa infected by $H$. $p y$ lori is shown in fig. $1 \mathrm{~b}$, lane 2 . After addition of the iNOS inhibitor 1400W (lane 3) and the COX-2 inhibitor NS-398 (lane 4), no significant difference was observed in the iNOS protein level when we compared bands of iNOS protein determined by densitometry.
The Western blot profile of COX-2 protein is shown in figure 2. In all patients without $H$. pylori infection and with normal mucosa (fig. 2 a, lane 2), COX-2 protein was undetectable and the addition of drugs did not modify the levels of COX-2. All patients with $H$. pylori infection and active gastritis showed a marked expression of COX-2 protein in antral biopsies (fig. $2 \mathrm{~b}$, lane 2). After addition of inhibitors of iNOS (lane 3 ) and inhibitors of COX-2 (lane 4), in the incubation medium, no difference was observed in the COX-2 protein level as determined by densitometry.

As shown in table 1, the gastric biopsy specimens of patients with $H$. pylori infection produced a significant increase in nitrite, after $24 \mathrm{~h}$ of incubation, compared with patients without $H$. pylori infection. This increase was significantly suppressed in $H$.-pylori-positive patients by addition of a selective iNOS inhibitor, $1400 \mathrm{~W}$, and also by that of a selective COX inhibitor, NS-398, while no difference was observed in the H.-pylori-negative patients. Measurement of $\mathrm{PGE}_{2}$ from $\mathrm{H}$.-pylori-positive samples revealed a significant increase in the capacity of this tissue to release PG compared with samples without $H$. pylori infection. Addition of an iNOS inhibitor, $1400 \mathrm{~W}$, had no significant effect on the concentration of $\mathrm{PGE}_{2}$, whereas the addition of NS-398 significantly inhibited the rise in the gastric mucosal concentration of $\mathrm{PGE}_{2}$. No difference was evident inside the H.-pylorinegative group.

\section{Discussion}

In the present study, we examined the cross-talk between products from iNOS and COX in human gastric biopsies during $H$. pylori infection by evaluating the effects of iNOS or COX inhibitors on levels of NO, $\mathrm{PGE}_{2}$ and protein expression of iNOS and COX. The data confirmed our previous results [19] that H.-pylori-positive patients experienced an increase in the levels of $\mathrm{NO}$ and $\mathrm{PGE}_{2}$ and an enhanced expression of iNOS and COX-2 proteins. It is reasonable to think that the high level of $\mathrm{PGE}_{2}$ released from the gastric mucosa of patients with $H$. pylori infection could limit the extent of mucosal damage caused by infection of $H$. pylori. The iNOS expression and the high release of $\mathrm{NO}_{2}$ ion that we have shown may play a role in the gastric inflammation associated with $H$. pylori infection and therefore in the pathogenesis of gastritis and peptic ulcer disease. Many lines of evidence have indicated that NO is a potent proinflammatory mediator and may have a multi- 


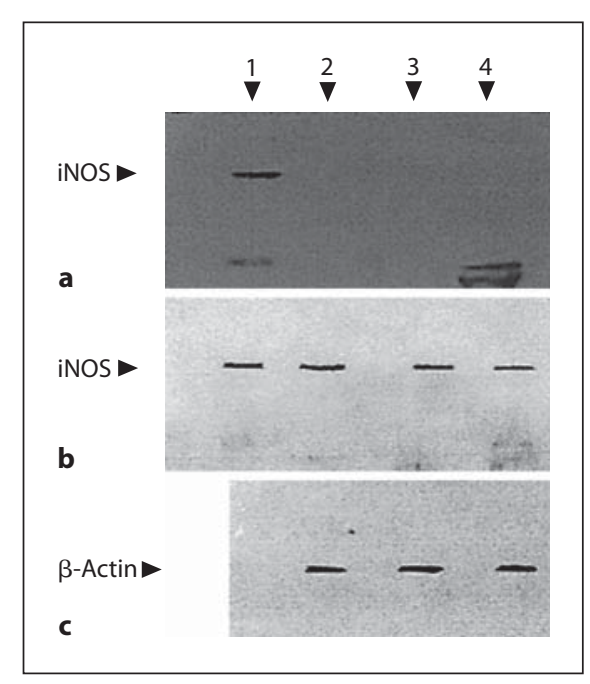

Fig. 1. iNOS protein level in human gastric biopsy. a The expression of iNOS protein was absent in mucosa of non-infected $H$. pylori patients (lane 2). After addition of the iNOS inhibitor 1400W $5 \mu \mathrm{M}$ (lane 3) and the COX-2 inhibitor NS-398 $10 \mu \mathrm{M}$ (lane 4), no expression of protein was evident. Lane 1 contains the iNOS standard. Similar results were obtained in all $H$.-pylori-negative patients. $\mathbf{b}$ The expression of iNOS protein was detected in all patients with $H$. pylori infection and with active gastritis (lane 2). Lane 3 indicates the effect of the inhibitor of iNOS, 1400W $5 \mu \mathrm{M}$, added to each specimen and incubated in the medium for $24 \mathrm{~h}$. Lane 4 indicates the effect of the inhibitor of COX-2, NS-398 10 $\mu \mathrm{M}$. Lane 1 contains the iNOS standard. Similar results were obtained in all the samples. c The iNOS signal was standardized against the corresponding $\beta$-actin signal. Bands corresponding to iNOS were quantitated by densitometry; no significant difference was observed in the expression of iNOS proteins.

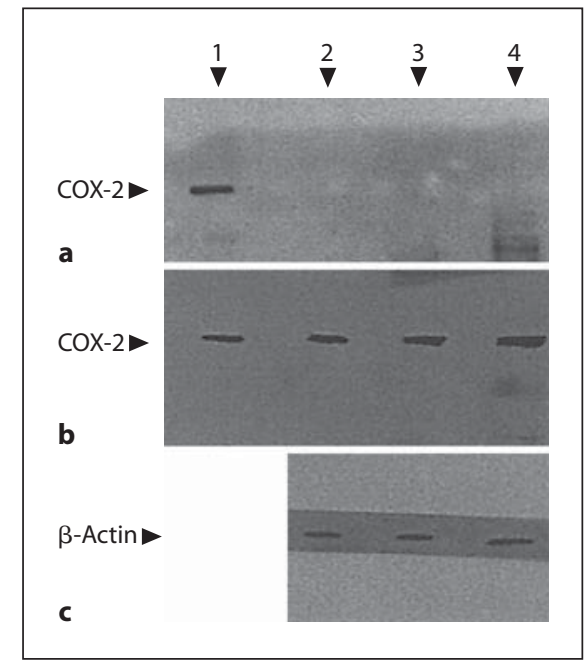

Fig. 2. COX-2 protein level in human gastric biopsy. a COX-2 protein expression was absent in all gastric mucosa of non-infected patients (lane 2). After addition of the iNOS inhibitor 1400W $5 \mu \mathrm{M}$ (lane 3) and the COX-2 inhibitor NS-398 $10 \mu \mathrm{M}$ (lane 4), no expression of proteins was evident. Lane 1 contains the COX-2 standard. Similar results were obtained in all $H$.-pylori-negative patients. $\mathbf{b}$ COX-2 protein expression was detected in all patients with H. pylori infection and active gastritis (lane 2). Lane 3 indicates the effect of the inhibitor of iNOS, 1400W $5 \mu \mathrm{M}$, added to each specimen and incubated in the medium for $24 \mathrm{~h}$. Lane 4 indicates the effect of the inhibitor of COX-2, NS-398 $10 \mu \mathrm{M}$. Lane 1 contains the COX-2 standard. Similar results were obtained from all samples. c The COX-2 signal was standardized against the corresponding $\beta$-actin signal. Bands corresponding to COX- 2 were quantitated by densitometry; no significant differences were observed.

Table 1. Levels of nitrite and PGs in antral gastric biopsy specimens

\begin{tabular}{|c|c|c|c|c|c|c|}
\hline H. pylori status & saline & $1400 \mathrm{~W}$ & NS-398 & saline & $1400 \mathrm{~W}$ & NS-398 \\
\hline Negative $(n=9)$ & $6.9 \pm 0.8$ & $6.2 \pm 0.8$ & $6.4 \pm 0.7$ & $763 \pm 61.3$ & $751 \pm 60.9$ & $698 \pm 59.8$ \\
\hline
\end{tabular}

Results are expressed as mean \pm SEM of patients. ${ }^{a} \mathrm{p}<0.01$ : significantly different from $H .-p y$ lori-negative patients. ${ }^{b} \mathrm{p}<0.01$ : significantly different $H$.-pylori-positive $1400 \mathrm{~W}$ and NS-398 versus saline. ${ }^{c} \mathrm{p}<0.01$ significantly different $H$.-pylori-positive NS-398 versus saline and 1400W.

faceted role in mutagenesis and carcinogenesis [20]. Therefore, aberrant or excessive expression of iNOS is often implicated in the oncogenesis and pathogenesis of cancer.

Improper activation or upregulation of iNOS or COX-2 has been shown to be associated with the patho- physiologies of certain types of human cancer as well as inflammatory disorders [21]. Since inflammation is closely linked to the promotion of certain tumours, substances with potent anti-inflammatory activities are anticipated to exert chemopreventive effects on carcinogenesis [20]. 
In this study, we found that the specific iNOS inhibitor 1400W suppressed the increase in NO level observed in gastric mucosa of all patients infected with $H$. pylori, but it did not have any effect on the increase in gastric mucosal $\mathrm{PGE}_{2}$ and on the expression of COX-2 and iNOS proteins. The selective COX-2 inhibitor NS-398 suppressed the upregulation in the gastric mucosal $\mathrm{NO}$ and $\mathrm{PGE}_{2}$ production that had been caused by infection of $H$. $p y$ lori although it exerted neither reduction of iNOS nor COX-2 at their protein levels.

Concerning the interaction of $\mathrm{NO}$ with COX activity, contradictory results have been reported on $\mathrm{NO}$ enhancing, inhibiting or having little effect on COX activity [22]. Here, we found that $1400 \mathrm{~W}$ prevented the formation of NO although this compound did not affect the accompanying rise in gastric mucosal $\mathrm{PGE}_{2}$ contents as a consequence of COX-2 activation. These results are not in accordance with the concept that iNOS-derived NO in inflammatory states may contribute to either induction or activation of COX-2. As for the cross-talk between iNOS and COX-2 and its products, including $\mathrm{PGE}_{2}$, we observed that a COX-2 inhibitor may modulate the iNOS pathway.

\section{Conclusion}

These findings suggest that COX-2 activity and/or its products may be closely related to the functional activation of iNOS but not to the expression of iNOS protein. The possible role played by COX activity in NO production in response to pathogens is interesting in the light of a possible pharmacological regulation of the beneficial or detrimental effects of NO.

\section{Acknowledgement}

This study was supported by the Ministero dell'Istruzione dell'Università e della Ricerca (grant 2007).

\section{References}

1 Tsutsumi S, Tomisato W, Takano T, Rokutan K, Tsuchiya T, Mizushima T: Gastric irritants induce apoptosis in guinea pig gastric mucosal cells in primary culture. Biochim Biophys Acta 2002;1589:168-180.

2 Konturek SJ: The physiological role of prostaglandins in cytoprotection; in Cheli R (ed): Gastric Protection. New York, Raven Press, 1988, p 13.

-3 Whittle BJR, Lopez-Belmonte J, Moncada S: Regulation of gastric mucosal integrity by endogenous nitric oxide: interactions with prostanoids and sensory neuropeptides in the rat. Br J Pharmacol 1990;99:607-611.

4 Moncada S, Palmer RMJ, Higgs EA: Nitric oxide: physiology, pathophysiology and pharmacology. Pharmacol Rev 1991;43:109142.

5 Dubois RN, Abramson SB, Crofford L, Gupta RA, Simon LS, Van de Putte LB, Lipsky PE: Cyclooxygenase in biology and disease. FASEB J 1998;12:1063-1073.

6 Fischer H, Becker JC, Boknik P, Huber V, Lüss H, Neumann J, Schmitz W, Domschke W, Stachura J, Kunturek JW: Expression of constitutive nitric oxide synthase in rat and human gastrointestinal tract. Biochim Biophys Acta 1999;1450:414-422.

-7 Garcia-Vitoria M, Garcia-Corchon C, Rodriguez JA, Garcia-Amigot F, Burrell MA: Expression of neuronal nitric oxide synthase in several types of the rat gastric epithelium. J Histochem Cytochem 2000;48: 1111-1119. $\checkmark 8$ Ambs S, Hussain SP, Harris CC: Interactive effects of nitric oxide and the p53 tumor suppressor gene in carcinogenesis and tumor progression. FASEB J 1997;11:443-448.

-9 Brown JF, Tepperman BL, Hanson PJ, Whittle BJR: Lipopolysaccharide induces $\mathrm{Ca}^{2+}$ independent nitric oxide synthase activity in rat gastric mucosal cells. Eur J Pharmacol 1994;292:111-114.

10 Franco L, Doria D: Nitric oxide enhances prostaglandin production in ethanol-induced gastric mucosal injury in rats. Eur J Pharmacol 1998;348:247-256.

11 Boughton-Smith NK, Evans SM, Laszlo F, Whittle BJR, Moncada S: The induction of nitric oxide synthase and intestinal vascular permeability by endotoxin in the rat. Br J Pharmacol 1993;110:1189-1195.

12 Tepperman BL, Soper BD: Nitric oxide synthase induction and cytoprotection of rat gastric mucosa from injury by ethanol. Can J Physiol Pharmacol 1994;72:1308-1312.

13 Wallace JL, Miller MJ: Nitric oxide in mucosal defense: a little goes a long way. Gastroenterology 2000;119:512-520.

14 Parsonnet J, Hansen S, Rodriguez L, Gelb AB, Warnke RA, Jellum E, Orentreich N, Vogelman JH, Friedmam GD: Helicobacter pylori infection and gastric lymphoma. $\mathrm{N}$ Engl J Med 1994;330:1267-1271.

15 Mannick EE, Bravo LE, Zarama G, Realpe JL, Zhang XJ, Ruiz B, Fontham ETH, Mera R, Miller MJS, Correa P: Inducible nitric oxide synthase, nitrotyrosine, and apoptosis in
Helicobacter pylori gastritis: effect of antibiotics and antioxidants. Cancer Res 1996;56: 3238-3243.

16 Dixon MF, Genta MG, Yardley JH, Pelay C: Classification and grading of gastritis. Am J Surg Pathol 1996;20:1161-1181.

17 Enzyme immunoassay prostaglandin E metabolite EIA kit. Ann Arbor, Cayman Chemical Company, 2004.

18 Bradford MM: A rapid and sensitive method for the quantitation of microgram quantitites of protein utilizing the principle of proteindye binding. Anal Biochem 1976;72:248-254.

19 Franco L, Talamini G, Carra G, Doria D: Expression of COX-1, COX-2, and inducible nitric oxide synthase protein in human gastric antrum with Helicobacter pylori infection. Prostaglandins Other Lipid Mediat 1999;58: 9-17.

20 Surh YJ, Chun KS, Cha HH, Han SS, Keum YS, Park KK, Lee SS: Molecular mechanisms underlying chemopreventive activities of anti-inflammatory phytochemicals: downregulation of COX-2 and iNOS through suppression of NF-kappa B activation. Mutat Res 2001;480-481:243-268.

21 Beyaert R (ed): Nuclear Factor кB: Regulation and Role in Disease. Dordrecht, Kluwer Academic Publishers, 2003.

22 Goodwing DC, Landino LM, Marnett LJ: Effects of nitric oxide and nitric oxide-derived species on prostaglandin endoperoxide synthase and prostaglandin biosynthesis. FASEB J 1999;13:1121-1136. 